\title{
A Comparative study of Proximity-Coupled Multiband Microstrip Antenna and Multimode Reduced Surface Wave Antenna
}

\author{
Pragati Mourya \\ M. Tech Scholar in Laxmi Narayan College of \\ Technology, Bhopal
}

\author{
Abhinav Bhargava \\ Assistant Professor in Laxmi Narayan College of \\ Technology, Bhopal
}

\begin{abstract}
This paper shows the comparative study of proximity-coupled feed, used for a multiband microstrip antenna (MMA) and multimode reduced surface-wave antenna based on the shorted-annular-ring reduced-surface-wave (SAR-RSW) element and the inverted-SAR-RSW ((ISAR-RSW) element. Microstrip antenna operates in four bands - WiMAX (3.3-3.7 GHz), LTE2300 (2300-2400 MHz), Bluetooth (2400-2485 $\mathrm{MHz}$ ), and WLAN (5.15-5.35 GHz, 5.725-5.825 GHz). Microstrip antenna has excellent radiation features along with a steady gain over the operating bands. Multimode reducedsurface-wave antenna has a reduced return-loss bandwidth of less than $10-\mathrm{dB}$. Its range of operation is $1.164-1.255$ and $1.552-1.610 \mathrm{GHz}$, a $3-\mathrm{dB}$ axial-ratio bandwidth from 1.16 to $1.26 \mathrm{GHz}$ and from 1.54 to $1.61 \mathrm{GHz}$. It produces a larger than 7-dBi gain in the entire operating band. To increase the impedance bandwidth, the multimode reduced-surface-wave antenna implements the proximity-coupled probe feeds. In order to restrain the mutual coupling between the SAR-RSW and ISAR-RSW elements, the defected ground structure bandrejection filters are implemented.
\end{abstract}

\section{Keywords}

Microstrip antenna, multiband, proximity feed, Microstrip antenna, Reduced surface wave element.

\section{INTRODUCTION}

In this era significant advancement in wireless communication needs antennas having superior performance, low weight and multiband operation. A microstrip patch antenna is a good option for all the above requirements. However, the microstrip antenna covers UMTS, LTE, Bluetooth, WiMAX, and WLAN bands. There are different antennas established to perform multiband operations. For example - planar monopole antenna, compact inverted-F antenna [5]. For instance [1]-[4], several strips are used in the radiating patches of a monopole to create many resonant modes. On the other hand, manufacture of such a 3-D structure for the inverted $\mathrm{F}$ antenna is difficult. Proximity coupling provides higher bandwidth and lower spurious radiation [6]. It also allows flexible feedline design. A proper feedline can be selected to agitate the preferred resonating modes and for the impedance matching. This paper includes the proximity-coupled multiband microstrip antenna and SAR-RSW antenna. In the direction of multiband responses slotted ground plane [7] is worn. By means of a meander microstrip feed it is agitated electromagnetically. In addition, a corner-truncated rectangular patch in the company of a rectangular slot at its center is worn, designed for impedance matching [8]

A Reduced-Surface-Wave (RSW) antenna belongs to a category of microstrip radiators designed to reduce the amount of surface-wave excitation $[9,10]$. The radiation pattern of the RSW antenna exhibits much lower levels of radiation down the horizon and toward the backside. This antenna is a superior entrant for high-precision GPS applications. An alternative RSW microstrip design is proposed in [11], referred as inverted-shorted-annular-ring reduced-surface- wave (ISAR-RSW) element. It has a compact planar dual-band RSW design. A dual-band GPSRSW antenna is proposed in [12], which has better radiation feat with smaller in magnitude in terms of volume and weight in accumulation, the mutual coupling flanked by the ISARRSW and SAR-RSW elements exists, and it has a great sway in a dual-band design for single-input feed network competent of concurrently feeding the SAR-RSW and ISAR-RSW elements [9]. In SAR-RSW and ISAR-RSW element defected ground structure (DGS) band-rejection filters and proximitycoupled probe feeds are adopted to increase bandwidth and restrain mutual coupling.

\section{DESIGN OF PROXIMITY-COUPLED MULTIBAND MICROSTRIP ANTENNA (PCMMA)}

The PCMMA antenna consists of a corner-truncated rectangular patch with a rectangular slot, meandered microstrip feed, and defected ground plane. The antenna is fabricated using 0.8-mm-thick FR4 substrate with a dielectric constant of 4.4 and has a small size of only $27 \times 24 \mathrm{~mm}$. A 3D sight of the PCMMA multiband antenna is shown in Fig. 1(a) and a picture of the fabricated antenna is shown in Fig. 1(b). The geometry of the projected multiband antenna by way of dimensions is shown in Fig. 1(c) and (d). FR4 substrate is used in two stacked layers, having a thickness of $0.8 \mathrm{~mm}$ each. Loss tangent of the substrate is 0.02 and the relative permittivity 4.4 . On the top of the upper layer a corner-truncated rectangular patch by means of a rectangular slot at its centre is printed. Bottom-side metal of this film is fully etched out. On top of the upper layer there is slotted ground structure and on the lower substrate layer a meandered microstrip feedline is printed to obtain proximity coupling. For connection of the inner conductor of an SMA connector to the microstrip feed, the length of the upper layer is slightly smaller than the lower layer.

Towards multiple resonators slots are etched in the ground plane of the lower layer having distinct resonance frequencies. The initial two resonance frequencies of a composed resonating slot whose one end is shorted and other end is opened are roughly the frequencies at which its length is $\lambda_{g} / 4$ $3 \lambda_{g} / 4$ and $\llbracket \lambda \rrbracket \mathrm{g}$. The resonance frequency can be tailored either by disturbing the slotline current distribution or changing its length. The dimension of the ground plane slots are optimized for the UMTS, LTE, and Bluetooth bands by straight microstrip feed as specified in Table I. 


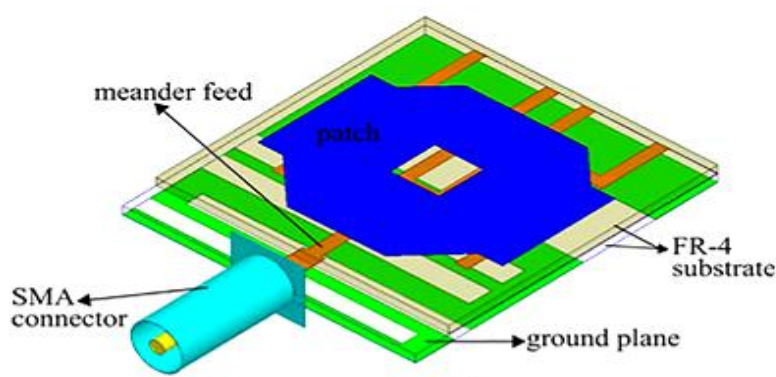

(a)

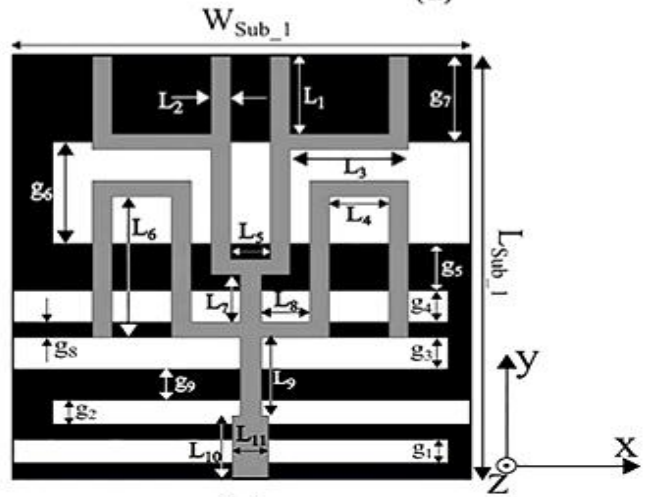

(c)
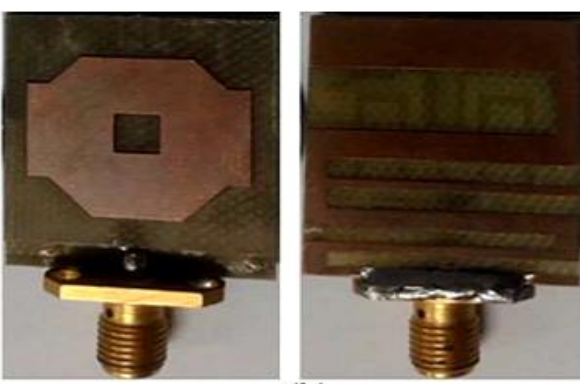

(b)

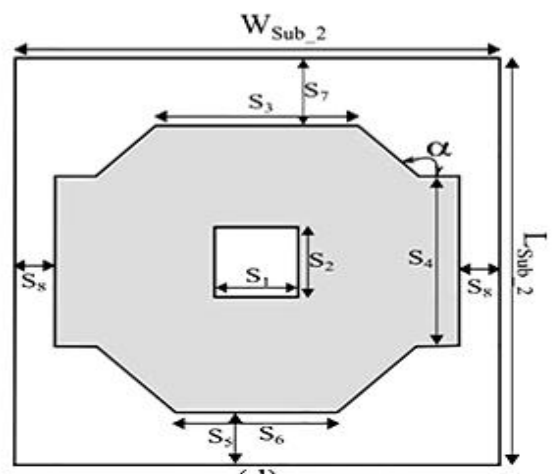

(d)

Fig. 1 Arrangement of the multiband-microstrip antenna: (a) 3-D vision, (b) image, (c) geometry of feedline and ground, and (d) upper patch geometry [8].

Table 1. Characterizing Parameter Values Of MultibandMicrostrip Antenna

\begin{tabular}{|c|c|c|c|c|c|c|c|c|c|}
\hline \multicolumn{2}{|c|}{ All dimension in mm } \\
\hline$L_{\text {Sub } 1}$ & $W_{\text {Sub } 1}$ & $L_{\text {Sub } 2}$ & $W_{\text {Sub } 2}$ & $L_{1}$ & $L_{2}$ & $L_{3}$ & $L_{4}$ & $L_{5}$ & $L_{6}$ \\
\hline 27 & 24 & 24 & 24 & 5 & 1 & 6 & 3 & 2 & 9 \\
\hline$L_{7}$ & $L_{8}$ & $L_{9}$ & $L_{10}$ & $L_{11}$ & $g_{1}$ & $g_{2}$ & $g_{3}$ & $g_{4}$ & $g_{5}$ \\
\hline 3 & 2.45 & 5 & 4 & 1.8 & 1. & 1. & 2 & 2 & 3 \\
& & & & & 5 & 5 & & & \\
\hline$g_{6}$ & $g_{7}$ & $g_{8}$ & $g_{9}$ & $S_{1}$ & $S_{2}$ & $S_{3}$ & $S_{4}$ & $S_{5}$ & $S_{6}$ \\
\hline 6.5 & 5.5 & 1 & 2 & 4 & 4 & 10 & 10 & 3 & 8 \\
\hline$S_{7}$ & $S_{8}$ & $\alpha$ & & & & & & & \\
\hline 4 & 2 & $135^{\circ}$ & & & & & & & \\
\hline
\end{tabular}

\section{MULTIMODE RSW ANTENNA}

\subsection{RSW Antenna Design}

The F4B substrate was used for fabrication of antenna with a loss tangent of 0.001 and dielectric constant of 2.65. The inner and outer radii are denoted as a and b correspondingly for the SAR element. The simulated radiation patterns with diverse SAR patch dimensions at resonant frequency of $1.58 \mathrm{GHz}$ are shown in Fig. The radiation patterns turn out to be narrower with the raise of $a$ and $b$. Thus, the patch dimensions of the SAR element are $\mathrm{a}=28.1 \mathrm{~mm}$ and $\mathrm{b}=55.7 \mathrm{~mm}$, to get superior low elevation characteristic.
The inner and outer radii are denoted as a_1 and b_1 correspondingly for the ISAR element. The beamwidths of the radiation patterns also turn out to be narrower with the raise of patch dimensions. A substitution is prepared between the antenna volume and low elevation characteristic. The ISAR element are a_ $1=74.0 \mathrm{~mm}$ and $b_{-} \_1=127.5 \mathrm{~mm}$ as its final magnitude, effect that the radiation outline of the ISAR element is broader than the SAR element for antenna volume drop. The SAR-RSW and ISAR-RSW elements were spaced $5^{\wedge \circ}$ and $\llbracket 10 \rrbracket^{\wedge \circ}$ apart respectively with $0.65 \mathrm{~mm}$ diameter. The antenna substrate and the ground are separated by $6 \mathrm{~mm}$ with air in between and the substrate height of antenna is $3 \mathrm{~mm}$. The F4B substrate is used to fabricate rectangle patches over the RSW patches of 1-mm thickness. The rectangle patches for the ISAR-RSW and SAR-RSW elements having dimensions of $6 \times 8$ and $2.6 \times 10 \mathrm{~mm}$, respectively. The SARRSW and the ISAR-RSW elements have a greater impedance matching from 1.552 to $1.610 \mathrm{GHz}$ to 1.164 to $1.255 \mathrm{GHz}$, correspondingly by means of the proximity-coupled probe feeds.

\subsection{Feed Network Design}

The SAR-RSW and ISAR-RSW elements is presented in [10], for a dual-band design with a single-input feed network which comprises a stub-line and 3-dB three-branch broadband hybrid combinations. The mutual coupling between the SARRSW and ISAR-RSW elements can be solved by using alternative feed network, which also delivers consistent 90 phase shifting and balanced power splitting to conserve the superior performance of RSW elements. The same F4B substrate is used as above, but $1.5 \mathrm{~mm}$ thickness, for designing and fabrication of the feed network as revealed in Fig. 2. 


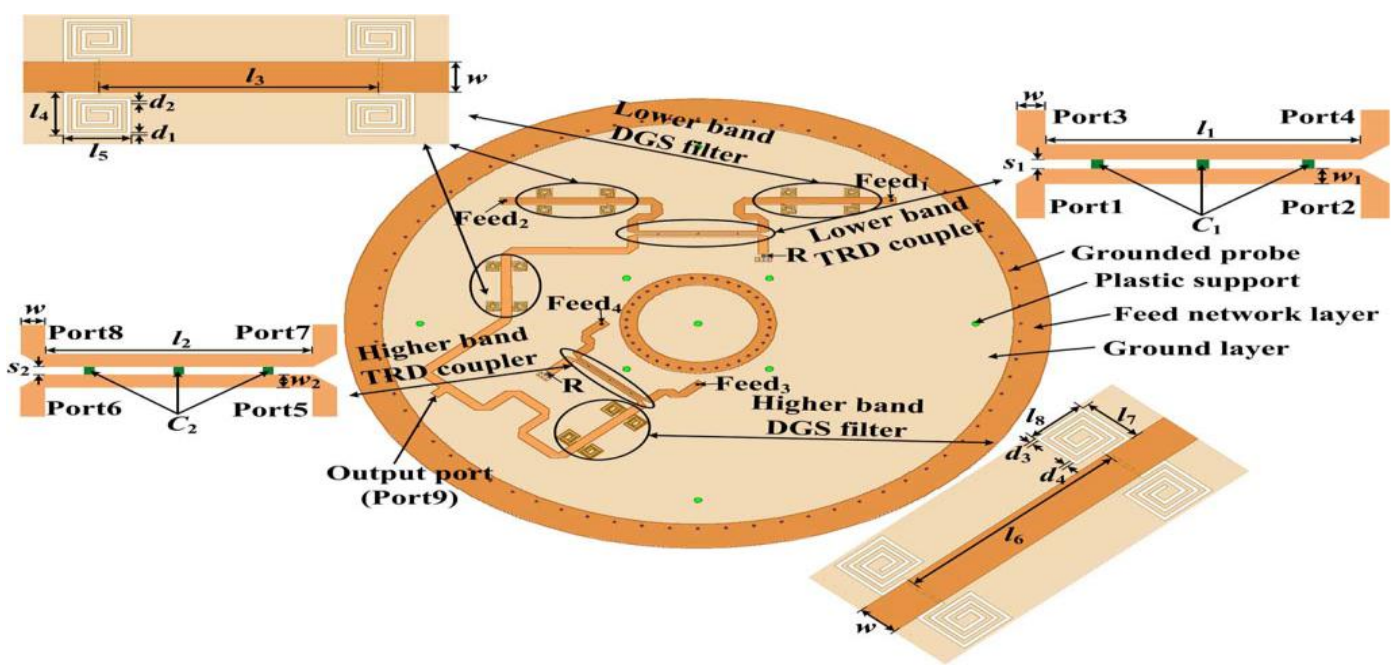

Fig. 2. Geometry of the feed network [9]

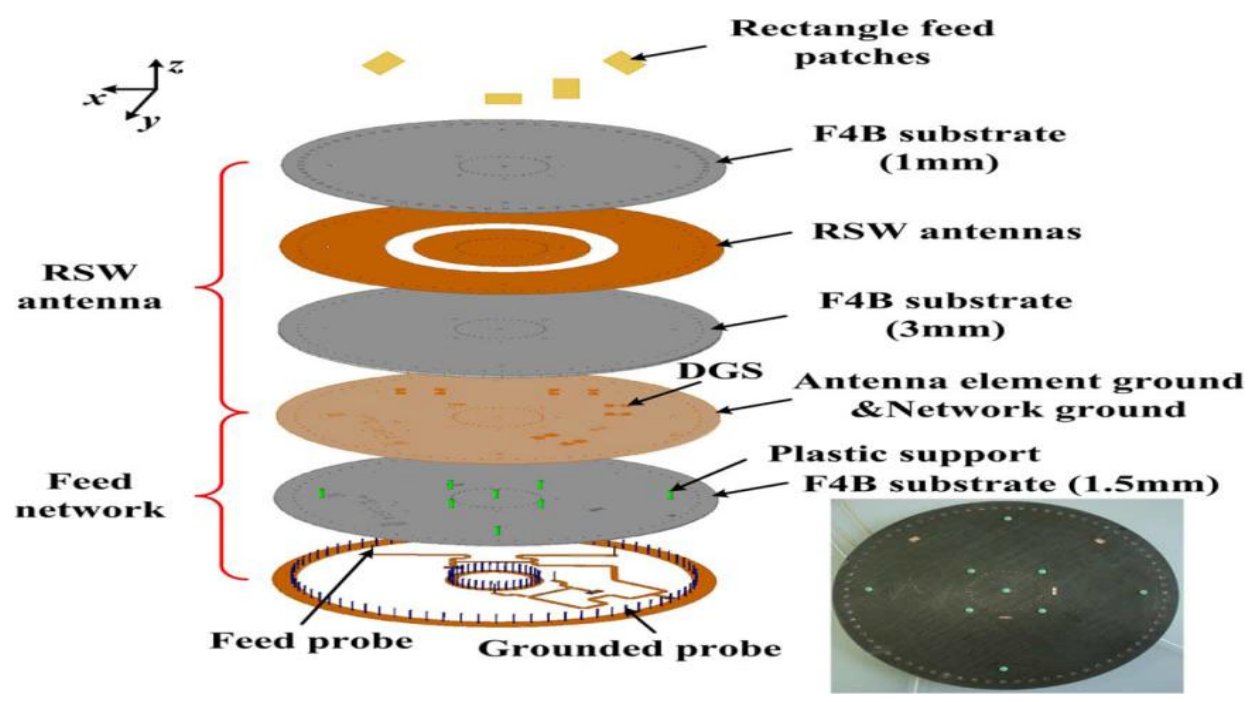

Fig. 3. (left) Exploded view and (right) prototype of the antenna [9]

TRD couplers are used in the direction of tight coupling to feed the SAR-RSW and ISAR-RSW elements. According to [11], number of the loaded coupled-line and the electrical length element are chosen to be 3 and 30, thus the odd - and even -mode characteristic impedances of the parallel coupled lines are $77.2 \Omega$ and $120.9 \Omega$, respectively. $C_{1}=2.2 \mathrm{pF}$ and $C_{2}=3 \mathrm{pF}$. The magnitude of the parallel coupled lines be: $w_{1}=$ $1.3 \mathrm{~mm}, w_{2}=1.3 \mathrm{~mm} s_{1}=1 \mathrm{~mm}, s_{2}=1 \mathrm{~mm}, l_{1}=7.9 \mathrm{~mm}$ and $l_{2}=6 \mathrm{~mm}$.

Higher-band DGS band-rejection filters are linked to the output ports of the lower-band TRD coupler with dimensions of $w=4.2 \mathrm{~mm}, l_{3}=24.6 \mathrm{~mm}, l_{4}=6 \mathrm{~mm}, l_{5}=6 \mathrm{~mm}$ $d_{1}=0.4 \mathrm{~mm}$,and $d_{2}=0.4 \mathrm{~mm}$, to restrain the mutual coupling between the ISAR-RSW and SAR-RSW element. The dimensions of the lower-band filter are as follows: $w=$ $4.2 \mathrm{~mm}, d_{3}=0.4 d_{4}=0.6 \mathrm{~mm}, l_{6}=24.6 \quad \mathrm{~mm}, l_{7}=7.6$ $\mathrm{mm}$, and $l_{8}=7.6 \mathrm{~mm}$.

The relation between return loss and reflection coefficient is defined by this relation as -

$$
\text { Return Loss }(d B)=-20 \log |\Gamma|
$$

\section{RESULT ANALYSIS}

The four bands of the multiband microstrip antenna as shown in Fig. 4, higher-order bands also seem at 4.12, 4.98, and 5.92 GHz. Next, two meander lines of length $L_{8}+2 L_{6}+L_{4}$ are presented on both sides of the traditional microstrip feed. The lines behave like a monopole antenna at $3.5 \mathrm{GHz}$.

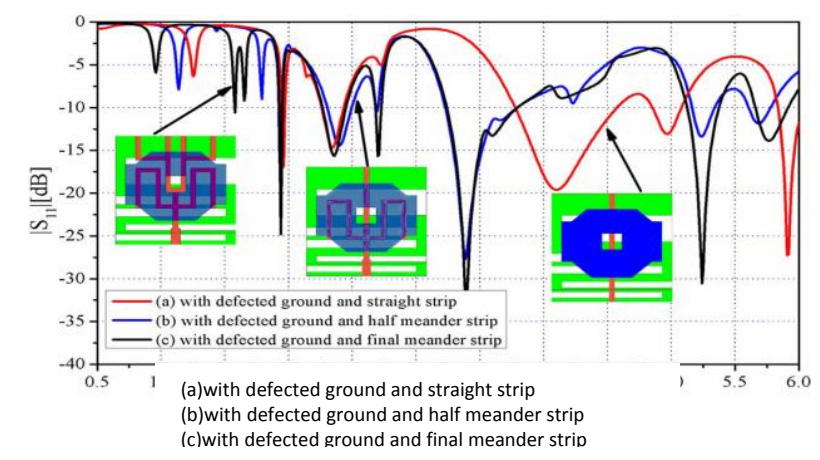

Fig. 4. Simulated result as reflection coefficient of multiband-microstrip antenna (a) with a straight strip and slotted ground, (b) with break out ground and halfmeander strip, and (c) with defected ground and final ambled strip [8] 


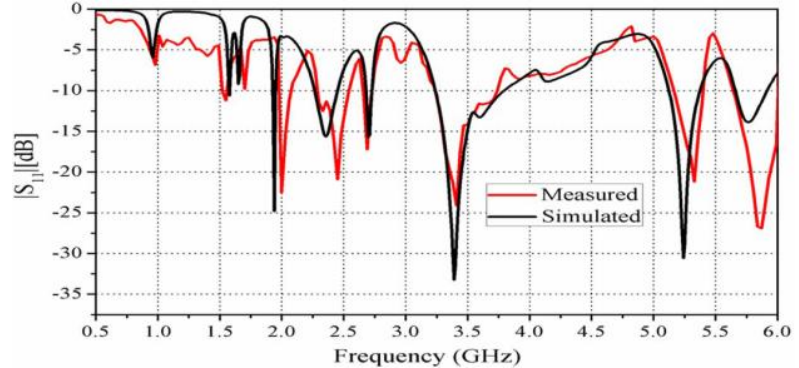

Fig. 5. Measured and simulated $\left|S_{11}\right|$.

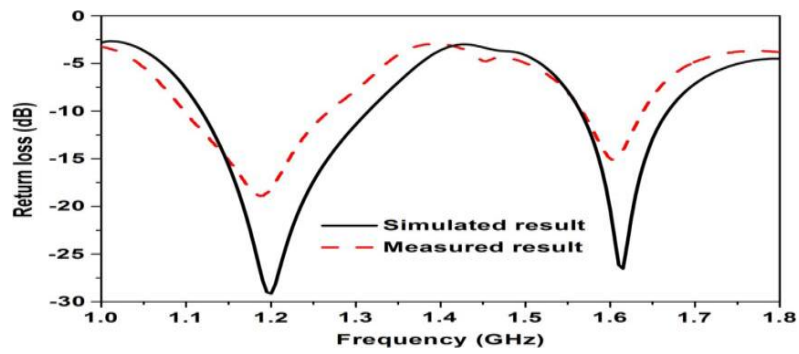

Fig. 6 Simulated and measured return loss of the feed network for MRSW antenna

It is observed that truncated corner and middle slot of the patch and two additional meander lines of length $L_{-}(1)+L_{-} 3$ of feed can be used for fine-tuning of input impedances simultaneously at all of the UMTS, LTE, Bluetooth, WiMAX, and WLAN bands. Fig. 6 shows the simulated and measured return loss of the Multimode Reduced Surface Wave (MRSW) antenna. The measured return loss is less than $0 \mathrm{~dB}$ from 1.164 to $1.225 \mathrm{GHz}$ and from 1.552 to $1.610 \mathrm{GHz}$. The righthand circular polarization (RHCP) fields are more than $20 \mathrm{~dB}$ with higher strength than the left-hand circular polarization (LHCP) fields by in the bore-sight direction. The gain of elevation is less than $17 \mathrm{~dB}$, which complements the ability of the antenna to reject multipath interference by polarization and low-level horizon. The reflection coefficient is measured using an Agilent N5242A vector network analyzer.

\section{RESULT ANALYSIS}

The four bands of the multiband microstrip antenna as shown in Fig. 4, higher-order bands also seem at 4.12, 4.98, and 5.92 GHz. Next, two meander lines of length $L_{8}+2 L_{6}+L_{4}$ are presented on both sides of the traditional microstrip feed. The lines behave like a monopole antenna at $3.5 \mathrm{GHz}$.

\section{CONCLUSION}

The configuration of small-size multiband microstrip antenna is presented that exhibits simultaneous four band operation at UMTS, LTE, Bluetooth, WiMAX, and WLAN bands. The multimode reduced-surface-wave antenna provides two bands of bandwidth $58 \mathrm{MHz}$ at L1 band and $91 \mathrm{MHz}$ at L2/L5 band is obtained. The multiband behavior has been achieved due to slotted ground plane and meandered microstrip feed in MMA. The radiation patterns are almost Omni-directional. The MMA is a compact antenna with more bands of operation. It is good for fabrication in industrial applications.

\section{REFERENCES}

[1] J. H. Yoon, Y. C. Rhee, and Y. K. Jang, "Compact monopole antenna design for WLAN/WIMAX triple- band operations," Microw. Opt. Technol. Lett., vol. 54, no. 8, pp. 1838-1846, Aug. 2012.

[2] J. H. Yoon and G. S. Kil, "Compact monopole antenna with two strips and a rectangular-slit ground plane for dual-band WLAN/WiMAX applications," Microw. Opt. Technol. Lett., vol. 54, no. 7, pp. 1559-1566, Jul. 2012.

[3] A. Mehdipour, A. R. Sebak, C. W. Trueman, and T. A. Denidni, "Compact multiband planar antenna for 2.4/3.5/5.2/5.8-GHz wireless applications," IEEE Antennas Wireless Propag. Lett., vol. 11, pp. 144-147, 2012.

[4] J. H. Lu and W. C. Chou, "Planar dual U-shaped monopole antenna with multiband operation for IEEE 802.16e," IEEE Antennas Wireless Propag. Lett., vol. 9, pp. 1006-1009, 2010.

[5] L. Pazin and Y. Leviatan, "Narrow-size multiband inverted-F antenna," IEEE Antennas Wireless Propag. Lett., vol. 10, pp. 139-141, 2011.

[6] C. A. Balanis, Antenna Theory. New York, NY, USA: Wiley, 2002.

[7] J. S. Hong and B. M. Karyamapudi, "A general circuit model for defected ground structure in plannar transmission lines," IEEE Microw. Wireless Compon. Lett., vol. 15, no. 10, pp. 706-708, Oct. 2005.

[8] Pritam Singh Bakariya, Santanu Dwari, Manas Sarkar, and Mrinal Kanti Mandal, "Proximity-Coupled Multiband Microstrip Antenna for Wireless Applications" IEEE Antennas And Wireless Propagation Letters, Vol. 14, 2015.

[9] Mohsen Koohestani, J.-F. Zürcher, Antonio A. Moreira, and Anja K. Skrivervik "A Novel, Low-Profile, Vertically-Polarized UWB Antenna for WBAN" IEEE Transactions on Antennas And Propagation, Vol. 62, No. 4, April 2014.

[10] D. R. Jackson, J. T. Williams, A. K. Bhattacharyya, R. L. Smith, S. J. Buchheit, and S. A. Long, "Microstrip patch designs that do not excite surface waves," IEEE Trans. Antennas Propag., vol. 41, no. 8, pp. 1026-1037, Aug. 1993.

[11] L. I. Basilio, J. T. Williams, D. R. Jackson, and R. L. Chen, "Characteristics of an inverted-shorted-annularring reduced-surface-wave antenna," IEEE Antennas Wireless Propag. Lett., vol. 7, pp. 123-126, 2008.

[12] L. I. Basilio, R. L. Chen, J. T. Williams, and D. R. Jackson, "A new planar dual-band GPS antenna designed for reduced susceptibility to low-angle multipath," IEEE Trans. Antennas Propag., vol. 55, no. 8, pp. 2358-2366, Aug. 2007.

[13] L. I. Basilio, "New GPS antennas designed for reduced multipath susceptibility," Ph.D. dissertation, Univ. Houston, Houston, TX, USA, 2003.

[14] C. I. Shie, J. C. Cheng, and S. C. Chou, "Transdirectional coupled-line couplers implemented by periodical shunt capacitors," IEEE Trans.Microw. Theory Tech., vol. 57, no. 12, pp. 2981-1988, Dec. 2009. 